\title{
ITINERARIOS INTERDISCIPLINARES EN EL GRADO DE EDUCACIÓN PRIMARIA
}

\section{INTERDISCIPLINAR ITINERARIES IN PRIMARy SCHOOL BACHELOR DEgREE}

Francisco Valverde Fernández*, Antonia Ramírez García, Manuel Mora Márquez, José Antonio López Fernández, Silvia Medina Quintana, José Carlos Arrebola Haro

*vde1vafef@uco.es

Universidad de Córdoba

Received: 30/06/2017_Accepted: 06/07/2018

\begin{abstract}
This paper is an interdisciplinary proposal that is being developed in the Bachelor Degree of Primary Education belonging to the Faculty of Education Sciences in the University of Córdoba, with the implication of two areas of knowledge: Didactics of Social Sciences and Didactics of Experimental Sciences. We implemented an educational itinerary through the city of Cordoba, in which the students had to prepare and explain several aspects of the different points of interest in the itinerary, regarding with Social and/or Experimental Sciences. The main objective of this activity is to make the students reflect on the importance of their surroundings and on the possibilities that Cordoba city offers to be used as an educational resource.
\end{abstract}

Keywords:Didactics of Social and Experimental Sciences; educational itinerary; transversality; interdisciplinarity.

\section{Resumen}

Este artículo se enmarca en una línea de trabajo iniciada por los integrantes del mismo en el Departamento de Didáctica de las Ciencias Sociales y Experimentales de la Facultad de Educación, en el grado de Educación Primaria. La realización de itinerarios didácticos interdisciplinares es una interesante herramienta didáctica en la formación del futuro profesorado. El objetivo principal del artículo consiste en poner de manifiesto el potencial del entorno inmediato y las posibilidades que la ciudad de Córdoba ofrece como recurso educativo. La metodología propone la participación de los estudiantes en todas las fases de trabajo, de un modo cooperativo y colaborativo. Los resultados ponen de manifiesto un mejor conocimiento del entorno a utilizar como recurso didáctico, vivencial y significativo.

Palabras clave: Didáctica de las Ciencias Sociales y Experimentales; Itinerario didáctico; Transversalidad; Interdisciplinariedad.

\section{INTRODUCCIÓN}

El presente trabajo se ha elaborado en colaboración entre las áreas de Didáctica de las Ciencias Experimentales y de las Ciencias Sociales de la Facultad de Ciencias de la Educación. Se trata de una secuencia didáctica que presenta como eje central la realización de un itinerario por el casco urbano de la ciudad de Córdoba, y en el que participan los diferentes grupos que componen el tercer curso del profesorado en formación del Grado de Educación Primaria (Medina y Otros, 2016; Medina y López, 2018).

Desde el ámbito educativo, el medio puede abordarse a través de numerosas materias, lo cual responde al tratamiento interdisciplinar que abordamos desde las áreas de conocimiento social y experimental. Resulta una obviedad que todos los acontecimientos sociales y naturales se producen en un lugar determinado. Por eso, el espacio cercano al alumnado puede devenir en una herramienta didáctica; no en vano, constituye el lugar donde se ponen de manifiesto los diferentes acontecimientos, hechos y fenómenos que constituyen las ciencias sociales y ciencias naturales.

Tratar e interactuar con el espacio puede realizarse desde edades tempranas mediante la Educación Física, con la realización de ejercicios de orientación, coordinación, etc.; en las Ciencias de la naturaleza, ya que todos los procesos ocurren en determinadas condiciones ecológicas y, por lo tanto, espaciales. Y, sobre todo, a través de las Ciencias Sociales, donde el ser humano utiliza el espacio y lo construye según sus intereses (el territorio). Por ello, se considera que las diferentes materias que están relacionadas con el espacio deben utilizar éste para enseñar a los y las estudiantes los diferentes procesos que ocurren en él, para que lo viva, lo comprenda, lo racionalice y lo localice; y, principalmente, para que entienda las diferentes interrelaciones que tienen lugar en él.

La formación didáctica es, pues, necesaria para dotar a los futuros y futuras docentes, en este caso de Educación Primaria, de los recursos, metodologías y enfoques que pongan de manifiesto un aprendizaje significativo y útil en la sociedad actual para comprender y actuar de forma justa y democrática (Santisteban y Pagès, 2007). 
El grado de innovación que se propone en este trabajo plantea dos niveles metodológicos de colaboración y cooperación, desarrollados en el apartado de objetivos. La secuencia didáctica programada pretende mostrar el trabajo cooperativo como herramienta para aprender el entorno cercano de forma integral e interdisciplinar.

\section{OBJETIVOS}

Los objetivos generales de este artículo se centran en dos líneas complementarias a nivel metodológico (figura 1).

En primer lugar, el desarrollo competencial del trabajo colaborativo entre el alumnado, fomentando la búsqueda y selección de información, así como su preparación para exponerla de forma adecuada al resto del grupo clase durante el desarrollo del itinerario. Itinerario que cuenta con una fase previa de preparación y otra fase final de afianzamiento y consolidación de los elementos aprehendidos. Asimismo, el alumnado trabaja diferentes aspectos relacionados con las materias de conocimiento, desarrolla capacidades y competencias de índole espacial, así como tratar cuestiones de carácter local, hasta ahora desconocidos, como elementos de la cultura y el patrimonio de la ciudad de Córdoba.

En segundo lugar, el trabajo de creación, articulación y desarrollo de la propuesta ha sido llevado a cabo por docentes pertenecientes a dos áreas diferentes, y que han coordinado las diferentes fases de la actividad.

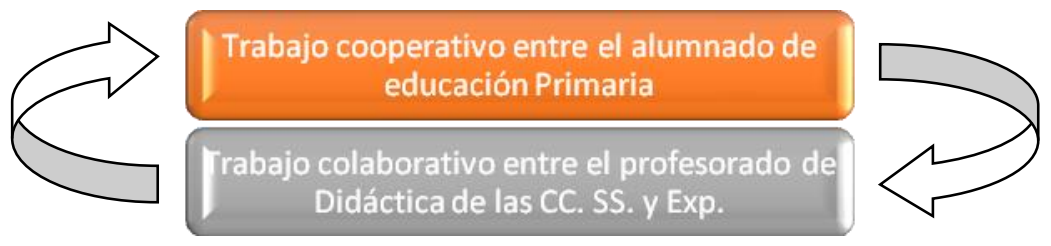

Figura 1: Objetivos planteados en la secuencia didáctica y relación entre los mismos

\section{MetodologíA}

Entre las principales herramientas pedagógicas que se pueden utilizar cuando se pretende salir del aula (Vilarrasa, 2003), están las visitas guiadas y, sobre todo, los itinerarios didácticos; muy enriquecedores cuando se trata de lugares urbanos (Valverde, Sequeiros y Loma, 2001; Martínez y García, 2008; Santolaria, 2014).

Como se viene indicando, la metodología propuesta en esta innovación está vertebrada por el trabajo colaborativo y cooperativo. En todo momento, se hace partícipe al alumnado para trabajar en pequeños grupos, interrelacionando con el resto del grupo clase. Los y las estudiantes participan preparando y buscando información así como exponiéndola, para finalizar trasladando y creando una nueva propuesta a su futuro alumnado de Educación Primaria.

El planteamiento sitúa al alumnado dentro de un enfoque activo. De este modo, los y las estudiantes vertebran el eje de las actuaciones, con la intencionalidad de motivar y propiciar un aprendizaje significativo, además de participativo.

Otro aspecto fundamental tenido en cuenta ha sido el trabajo por competencias. Teniendo en cuenta las establecidas por el Ministerio de Educación para la etapa de Educación Primaria, la secuencia fomenta del trabajo y desarrollo de varias capacidades y competencias, como se indicará posteriormente.

Igualmente, conviene resaltar respecto a la metodología su carácter flexible, adaptada a las circunstancias de cada grupo y de cada parada, adecuando el planteamiento inicial al discurrir de la actividad.

\section{DESCRIPCIÓN}

La secuencia didáctica que se programa gira en torno a las diferentes fases que conlleva la puesta en marcha de un itinerario interdisciplinar. El que plantea en este artículo, teniendo en cuenta el nivel universitario del alumnado al que iba dirigido (dos grupos de clase que suman un total de 120 estudiantes), implementa una metodología que ha partido de la base de la integración y la interdisciplinariedad entre la Didáctica de las Ciencias Sociales y de las Ciencias Experimentales.

Además, se tiene en cuenta que es el alumnado, en grupos de cuatro o cinco, el encargado de preparar la exposición de las paradas, previamente establecidas por el profesorado y donde se deben abordar los objetivos establecidos por este.

En la figura 2 se comenta la planificación seguida en el desarrollo de nuestra secuencia. De esta forma, el primer paso consiste en que el profesorado exponga, en una sesión teórica, la actividad-itinerario con las correspondientes paradas, y se configuran los grupos de trabajo. 


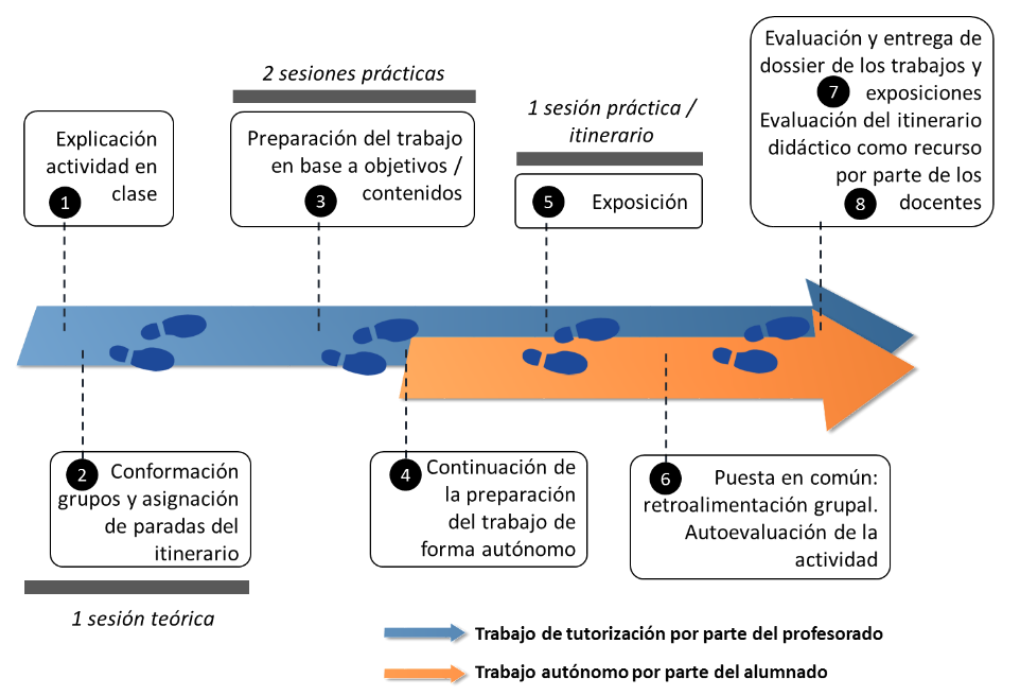

Figura 2: Planificaciones y sesiones de trabajo de la secuencia didáctica

La preparación de las paradas asignadas a cada grupo conlleva dos sesiones prácticas, donde se abordan desde la descripción de la parada hasta las referencias bibliográficas citadas en el dossier final, pasando por la realización de mapas conceptuales, esquemas y demás recursos infográficos. También se muestra el aparato teórico del desarrollo de un itinerario didáctico; sus principales funciones (como recurso motivador, integrador, evaluador, etc.), además de tener en cuenta los momentos en los que se puede dividir el itinerario (antes, durante y después), así como las diferentes metodologías que se pueden llevar a cabo en su desarrollo.

A partir de esta sesión, y hasta la propia realización del itinerario, cada grupo de trabajo prepara un dossier-memoria, donde se deben plasmar los aspectos más relevantes de la parada seleccionada o asignada. La evaluación final, tanto de las memorias como del potencial recurso didáctico, supone la última fase de la planificación. Todo el proceso esta convenientemente tutorizado por parte del profesorado responsable.

La planificación de lugares seleccionados para ubicar las paradas, así como los objetivos planteados permiten trabajar aspectos relacionados con la historia (la evolución histórica, la huella de las distintas culturas, las manifestaciones de las diversas sociedades, etc.), contenidos de geografía urbana que caracterizan la parada (antiguos espacios industriales, planificación del tranvía, reutilización del patrimonio, nuevas áreas residenciales, etc.), así como cuestiones relacionadas con las ciencias experimentales (los medios de transporte y locomoción; el funcionamiento de los hornos de fábricas; el uso del agua desde un punto de vista tecnológico, etc.) que, en un futuro, pueden servirles en las prácticas en sus clases de Educación Primaria.

El profesorado, a partir de aquí, ejerce de guía. Es tarea del alumnado preparar la información representativa de cada parada, buscar la información relativa a los elementos más importantes y característicos desde el punto de vista social, histórico, espacial o natural. Además, deben organizar la exposición in situ de las peculiaridades del mismo.

El resultado es un itinerario con siete paradas (variable en función de los grupos de trabajo confeccionados) en ubicaciones emblemáticas de la ciudad. Además, se ha tenido en cuenta la experiencia sobre las peculiaridades de la ciudad así como las recomendaciones de algunos investigadores que, como Insa (2002) recomiendan no incluir un elevado número de paradas, buscar ubicaciones con poco tráfico y que estén relativamente cercanas.

\section{RESULTADOS OBTENIDOS}

Los resultados de la puesta en práctica de esta secuencia didáctica, que requeire varios meses de trabajo coordinado, muestran el cumplimiento de los objetivos propuestos en el planteamiento inicial. La actividad coordinada entre las diferentes áreas se muestra como ejemplo de trabajo colaboración en la formación docente universitaria, reflejado por la motivación y finalidad señalada por el alumnado, así como el interés de la participación de otras áreas de conocimiento.

La coordinación entre profesorado y alumnado, así como la evaluación, tanto del proceso de enseñanza como de aprendizaje, se refleja, además, en la elaboración de materiales didácticos. En este aspecto, la plataforma Moodle se muestra como herramienta de conexión virtual permanente para el envío de archivos entre profesorado y alumnado, resolución de dudas, elaboración de materiales, etc.

Los resultados de la evaluación, así como la percepción y opiniones mostradas por el alumnado en un cuestionario de satisfacción (Figura 3), permiten constatar el funcionamiento efectivo de la secuencia didáctica, sobre todo al potenciar la 
toma de decisiones y la mejora de la capacidad crítica. También se promueve la búsqueda y preparación de la información, así como la exposición oral al resto del grupo clase.

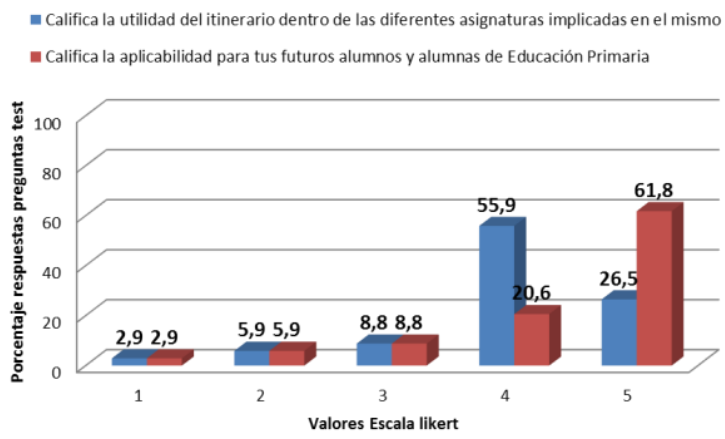

Figura 3: Valoración de la propuesta didáctica por parte del alumnado

Respecto a las competencias, este planteamiento propicia el escenario ideal para el aprendizaje por pares y el trabajo por competencias, tal como se señala a continuación:

- Competencia en comunicación lingüística. El fomento de esta competencia es claro, ya que los distintos grupos, además de preparar y llevar a cabo una exposición para elaborar cada punto del itinerario, deben elaborar un dossier sobre éste. Además, uno de los grupos era el perteneciente al itinerario bilingüe, por lo que se potenciaron las competencias en lengua inglesa.

- Competencia matemática y competencias básicas en ciencia y tecnología. Fundamentalmente se fomentan las segundas, ya que algunos de los contenidos a desarrollar en las paradas están relacionados con las ciencias experimentales y/o la tecnología, como el vehículo eléctrico o la locomotora de vapor.

- Competencia digital. En una actividad como ésta, en la que los alumnos y alumnas deben buscar información sobre diferentes aspectos indicados por el profesorado, resulta fundamental la búsqueda de ésta en internet, junto a la bibliografía recomendada. Además, en la fase de preparación se plantea la utilización de visores cartográficos online para preparar las diferentes paradas, así como el conjunto del itinerario, también desde una doble perspectiva: como herramienta de información útil para este cometido y como recurso didáctico para la formación del profesorado.

- Aprender a aprender. De manera complementaria a la anterior competencia, el alumnado debe ser competente para buscar información sobre aspectos propuestos por el profesorado y diferenciando la que es útil de la que no lo es. Además, se debe sintetizar todo aquello que se encuentra, pues el tiempo dado para su exposición es limitado. Igualmente, es necesario contemplar el modo con el que se va a realizar la exposición, elemento también contemplado para la evaluación.

- Competencias sociales y cívicas. Además del respeto en una actividad colaborativa como esta, el itinerario por la ciudad permite evidenciar diferentes situaciones sociales como desigualdades, conflictos, el respeto o destrucción del patrimonio, etc.

- Conciencia y expresiones culturales. De manera análoga a como sucede con las Ciencias Experimentales, la segunda disciplina puesta en alza en esta actividad son las Ciencias Sociales, ya que todos los grupos trabajan un tema relacionado con esta disciplina, bien Geografía, Historia o Historia del Arte. La ciudad de Córdoba (Figura 4) ofrece un marco incomparable para tratar la conciencia histórica. Las diferentes manifestaciones culturales, reflejo de los pueblos que han habitado la ciudad, permiten trabajar el patrimonio cultural e histórico a lo largo del entramado urbano, lo que permite trasladar la singularidad e importancia de construcciones y monumentos históricos.

Esta secuencia didáctica resulta de interés para el alumnado, quienes valoran la originilidad de la propuesta y confirman su valor motivacional. A tenor de la recogida de evidencias, se valora de forma positiva la coordinación horizontal de las asignaturas, considerandolo, por tanto, una buena práctica docente, repetible en otras asignaturas del curso y del Grado. Tanto desde el punto de vista de los contenidos como de la organización, este planteamiento supone un avance en la consolidación de actividades programadas y coordinadas que ofrecen una visión global e interdisciplinar, además de centralizar y unificar el trabajo del alumnado. 


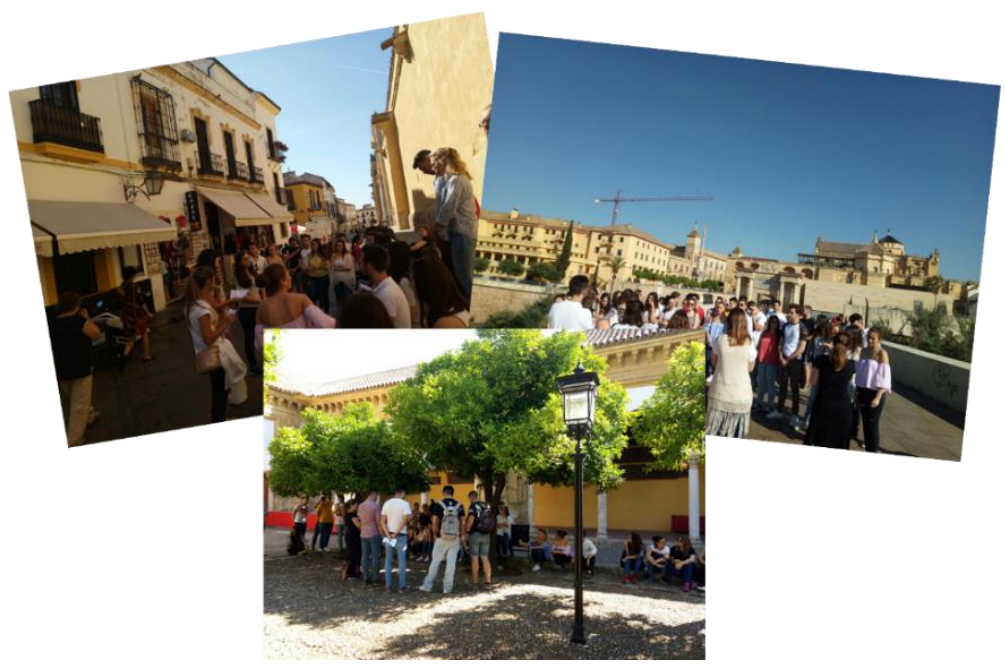

Figura 4: Diferentes situaciones, a modo de ejemplo de las paradas y exposiciones en los itinerarios didácticos realizados (alrededores e interior de la Mezquita-Catedral de Córdoba)

\section{CONCLUSIONES Y DISCUSIÓN}

Con este trabajo se quiere mostrar una metodología docente fruto de la colaboración entre dos áreas universitarias (Didáctica de las Ciencias Sociales y Didáctica de las Ciencias Experimentales) que comparten el mismo alumnado en el Grado de Educación Primaria donde, como se ha visto, se puede poner en marcha un trabajo teórico-práctico en relación al trabajo fuera del aula.

La secuencia didáctica que se propone plantea la elaboración de un itinerario didáctico, donde el profesorado solo indica los puntos de parada en la ciudad de Córdoba y la temática a trabajar; los y las alumnas, por su parte, en grupos de cuatro o cinco, se encargan de buscar y seleccionar la información, preparar los materiales y exponer in situ en una de las paradas seleccionadas. De este modo, a través de una metodología activa, el alumnado en formación participa en el proceso de elaboración de un itinerario, desarrollando competencias y capacidades que, en un futuro, deberá poner en funcionamiento.

Esta metodología permite fomentar la transversalidad y la cooperación entre diferentes áreas en la docencia universitaria, en este caso integradas en el mismo departamento. El desarrollo de esta actividad ha requerido de la puesta en común de objetivos y contenidos, a través de varias reuniones de trabajo, que luego se trasladan a las clases prácticas con el alumnado, donde éstos han trabajan en la búsqueda de información y en su presentación. Asimismo, la realización de un itinerario didáctico como éste permite trabajar distintas competencias, no sólo las más cercanas a las materias.

Así, además de las competencias propias de las materias disciplinares de carácter didáctico social y experimental, se abordan las competencias digitales y de aprender a aprender mediante el trabajo de búsqueda de información por parte del alumnado, así como las competencias lingüísticas -incluidas en otro idioma, en el caso del itinerario bilingüe- y sociales y cívicas a través de las vivencias y experiencias que se plantean a lo largo y ancho del entramado urbano.

En definitiva, con esta propuesta se ha pretendido trabajar con el alumnado una serie de contenidos interdisciplinares y potenciar una serie de competencias al tiempo que se le hacía partícipe de la capacidad de la ciudad de Córdoba como herramienta didáctica. 


\section{BIBLIOGRAFÍA}

INSA, Y. Itinerarios urbanos, recursos y materiales didácticos para explicar la ciudad. En Íber didáctica de las ciencias sociales: geografía e historia, 32, 2002, 89-95.

MARTÍNEZ, F. J.; GARCÍA, A. J. Itinerarios didácticos por fuente álamo (Murcia), una estrategia educativa de innovación en el proceso de enseñanza aprendizaje en educación secundaria. En Espiral cuadernos del profesorado, 1, 2008, 1-9.

Medina, S.; ArrebolA, J.C.; MOrA, M.; LópeZ, J.A. Propuesta de itinerario interdisciplinar en la formación del profesorado de Educación Primaria en el ámbito de las Ciencias Sociales y Experimentales. En Didáctica de las Ciencias Experimentales y Sociales, 31, 2016, 79-98.

MEDiNA, S.; LÓPEZ, J.A. El patrimonio en la formación del profesorado en Educación Infantil: una propuesta activa a través del casco histórico de Córdoba. En López, E.; García, C.R.; Sánchez, M. (Eds.) Buscando formas de enseñar: investigar para innovar en didáctica. Valladolid, 2018.

RD 126/2014, de 28 de febrero, por el que se establece el currículo básico de la educación primaria.

SANTOLARIA, A. La ciudad como recurso didáctico. En Edetania, 45, 2014, 235-244.

VAlverde, F.; SEQUeIros, C. y LOMA, M. La ciudad y el patrimonio urbano como forjadores de identidad. En EsTEPA, FRIERA y PIÑEIRO. Identidades y territorios: un reto para la didáctica de las ciencias sociales, 579-586. Oviedo, 2001.

VILARrasA, A. Salir del aula. En Íber. Didáctica de las ciencias sociales, geografía e historia, 36, 2003, 5-6.

- Salir del aula. Reapropiarse del contexto. En Íber. Didáctica de las ciencias sociales, geografía e historia, 36, 2003, $13-25$.

\section{OTRAS OBRAS CONSULTADAS}

Cortés, A. M. La interdisciplinaridad en la educación universitaria. En Anuario del CIJS. Anuario n¹0, sección 4, 2007, 401415. [en línea]. www.derecho.unc.edu.ar/ publicaciones/anuarios-del-cijs.../ anuario.../file. fecha de consulta: 2 de febrero de 2016

HernÁndeZ, F. X. Sociedad, patrimonio y enseñanza. Estrategias para el siglo XXI. En GONZÁLEZ GALLEGO, I. (dir.). En La geografía y la historia, elementos del medio, 245-277. Madrid, 2002.

LÓPEZ, F.; SEGURA, J. Los itinerarios didácticos: un recurso interdisciplinar y vertebrador del currículum. En Espiral cuadernos del profesorado. Revista digital del centro de profesorado cuevas-olula. Vol. 6, 2013, 12. disponible en: http://www. cepcuevasolula.es/espiral.

MÉndeZ, A., Sierra, B. y MAÑANA, J. Percepciones y creencias de los docentes de primaria del Principado de Asturias sobre las competencias básicas. En Revista de educación, 362, septiembre-diciembre 2013, 737-761.

MOYA, J. Las competencias básicas en el diseño y el desarrollo del currículum. En Revista qurriculum, 21, 2008, 57-78. [en línea]. Disponible en: http://revistaq.webs.ull.es/ anteriores/numero21/moya.pdf. Fecha de consulta: 2 de febrero de 2016

OCDE (2001). Definition and selection of competencies: theoretical and conceptual foundations (deseco). 11 feb 2016, de OCDE sitio web: http:// www.oecd.org/edu/skills-beyondschool/41529556.pdf

OlAVE, D. El itinerario didáctico: una propuesta metodológica para el análisis geo-histórico local. En Geoenseñanza, 10, 2005, 197-208.

RuBIO, P. Itinerario didáctico por la sierra de Albarracín. En Serie geográfica, 11, 2012, 101-116.

RUIZ, C.; PALACIO, I. Higienismo, educación ambiental y previsión escolar. Antecedentes y prácticas de educación social en España (19001936). Valencia, 1999. 
SÁNCHEZ, A. El trabajo de campo y las excursiones. En Enseñar geografía. De la teoría a la práctica. Madrid, 1995.

SAntisteban, A.; PAgÉs, J. La educación democrática de la ciudadanía. Una propuesta conceptual. En ÁviLA, R. Mª; LÓPEZ, J. R.; FERNÁNDEz, E. (eds.), Las competencias profesionales para la enseñanza aprendizaje de las ciencias sociales ante el reto europeo y la globalización, 353-368. Bilbao, 2007. 